\title{
Defect-rich ZnO nanosheets of high surface area as an efficient visible-light photocatalyst
}

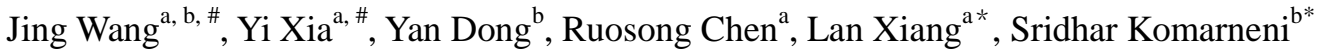 \\ ${ }^{a}$ Department of Chemical Engineering, Tsinghua University, Beijing, 100084, China \\ ${ }^{\mathrm{b}}$ Department of Ecosystem Science and Management and Materials Research Institute, Materials \\ Research Laboratory, The Pennsylvania State University, University Park, PA 16802, USA
}

\begin{abstract}
A facile ultra-rapid solution method was developed to fabricate $\mathrm{ZnO}$ nanosheets with tunable BET surface area and rich oxygen-vacancy defects. The addition of $1 \mathrm{~mol} \cdot \mathrm{L}^{-1} \mathrm{Na}_{2} \mathrm{SO}_{4}$ led to an increase of BET surface area of $\mathrm{ZnO}$ nanosheets from 6.7 to $34.5 \mathrm{~m}^{2} / \mathrm{g}$, through an electrostatic-controlled growth and self-assembly mechanism. Detailed analysis based on Raman scattering, room-temperature photoluminescence, X-ray photoelectron spectroscopy and electron spin resonance revealed that the as-prepared $\mathrm{ZnO}$ nanosheets were rich in oxygen-vacancies. Increased BET surface area led to a further increase of surface oxygen-vacancy concentration. The rich oxygen-vacancies promoted the visible-light absorption of the $\mathrm{ZnO}$ nanosheets, leading to high photocurrent responses and photocatalytic activities towards the degradation of rhodamine B (apparent rate constants, $\left.\mathrm{k}=0.0179 \mathrm{~min}^{-1}\right)$ under visible-light illumination $(\lambda>420 \mathrm{~nm})$, about 13 and 11 times higher, respectively than that of $\mathrm{ZnO}$ nanoparticles with few oxygen defects. In addition, the high-surface-area $\mathrm{ZnO}$ nanosheets could be effectively hybridized with $\mathrm{Ag}_{3} \mathrm{PO}_{4}$ nanoparticles, resulting in a further enhancement of the visible-light photocatalytic performance $\left(\mathrm{k}=0.0421 \mathrm{~min}^{-1}\right)$.

\# These two authors contributed equally.

* Corresponding authors. Email: xianglan@mail.tsinghua.edu.cn (L.Xiang); sxk7@psu.edu (S. Komarneni);
\end{abstract}

(C) 2016. This manuscript version is made available under the Elsevier user license http://www.elsevier.com/open-access/userlicense/1.0/ 
This increase in performance was attributed to the increased visible-light absorption as well as the energy level matching, the latter leading to efficient charge transfer between oxygen-vacancy-rich $\mathrm{ZnO}$ nanosheet and $\mathrm{Ag}_{3} \mathrm{PO}_{4}$, suggesting a synergistic effect of surface oxygen vacancies and $\mathrm{Ag}_{3} \mathrm{PO}_{4}$ coupling.

Keywords: $\mathrm{ZnO}$; defect-rich; high surface area; visible-light photocatalyst; synergistic effect

\section{Introduction}

Semiconductor photocatalysis is a promising solution for many environment-related issues. Among various oxide semiconductor photocatalysts, $\mathrm{ZnO}$ has been intensively studied for degradation of organic pollutants due to its high quantum efficiency, nontoxic nature and low cost [1]. Unfortunately, intrinsic $\mathrm{ZnO}$ can only absorb ultraviolet light (5-7\% of the total sunlight [2]) due to its wide band gap (3.2 eV) [3], which limits the photocatalytic efficiencies for practical applications. Research is now mainly focused on developing $\mathrm{ZnO}$ photocatalysts with high visible-light driven photo-activities via band-gap engineering. For example, doping of $\mathrm{ZnO}$ with various non-metal or transit-metal ions would make $\mathrm{ZnO}$ absorb photons of lower energy by generating narrower impurity bands [4-6]. Compared with the conventional doping methods, introducing oxygen-related defects into $\mathrm{ZnO}$ could also enhance the visible-light absorption, by creating defect states lying under the conduction band (CB) of $\mathrm{ZnO}$ without introducing any impurities, providing an effective approach for visible-light photocatalysis [7, 8]. Oxygen defect-rich $\mathrm{ZnO}$ was synthesized previously by chemical vapor deposition [9], plasma treatment [10], high-temperature annealing in atmospheres (e.g. $\mathrm{H}_{2}$, Ar) [11] and thermal decomposition of specific precursors (e.g. $\mathrm{ZnO}_{2}$ ) [8]. Besides the above methods 
involving complicated procedures, there have been a few reports on the formation of $\mathrm{ZnO}$ nanostructures rich in oxygen defects by solution methods $[12,13]$, which are more attractive because of mild conditions and low energy consumption. Therefore, it is highly desirable to develop simple and controllable solution synthesis of oxygen defect-rich $\mathrm{ZnO}$ nanostructures.

The visible-light photocatalytic process strongly depends on the surface properties of oxide photocatalysts $[14,15]$. Only the oxygen defects on the surface could serve as charge carrier traps where the charges transfer to the adsorbed species and prevent the photogenerated electron/hole $\left(\mathrm{e}^{-} / \mathrm{h}^{+}\right)$ recombination while bulk defects only act as charge carrier traps where $\mathrm{e}^{-} / \mathrm{h}^{+}$recombine during photocatalytic process [16]. A correlation of photocatalytic activity with the concentration of surface defects has been observed for $\mathrm{TiO}_{2}$ nanocrystals [14, 17]. Therefore, construction of an oxide photocatalyst with high surface to volume ratio would be beneficial to increase the content of surface oxygen defects, which prevent the $\mathrm{e}^{-} / \mathrm{h}^{+}$recombination and improve the visible-light photocatalytic activity. Although the relationship between oxygen defects and visible-light photocatalytic performance of $\mathrm{ZnO}$ nanostructures was widely investigated [18-21], little or no work addressed the surface area effects in visible-light photocatalysis. The surface areas of the $\mathrm{ZnO}$ nanostructures used in the above studies were generally small $\left(<10 \mathrm{~m}^{2} / \mathrm{g}\right)$ or they were not reported. For example, the visible-light photocatalytic properties of $\mathrm{ZnO}$ tetrapods [18] and $\mathrm{ZnO}$ rods [12] were found to be induced by oxygen vacancies, however, the BET surface areas of the tetrapods and rods were only 2.2 and $7.2 \mathrm{~m}^{2} / \mathrm{g}$, respectively. Inspired by the above studies, the synthesis of $\mathrm{ZnO}$ nanostructures of high surface area and rich in oxygen defects could be an effective strategy to improve the visible-light photocatalytic performance. 
In this work, we present a facile ultra-rapid solution synthesis of $\mathrm{ZnO}$ nanosheets with high BET surface area along with rich oxygen-vacancy defects. The BET surface area of $\mathrm{ZnO}$ was simply tuned by salt-addition, through an electrostatic-controlled self-assembly mechanism. Because of the rich oxygen-vacancies, the visible-light photocurrent responses and photocatalytic activities of the $\mathrm{ZnO}$ nanosheets were significantly improved compared to $\mathrm{ZnO}$ nanoparticles with few oxygen defects. Additionally, the hybridization of $\mathrm{ZnO}$ nanosheets with $\mathrm{Ag}_{3} \mathrm{PO}_{4}$ nanoparticles resulted in higher photocatalytic activities owing to the synergistic effect of surface oxygen vacancies and $\mathrm{Ag}_{3} \mathrm{PO}_{4}$ coupling. The mechanisms for enhanced visible-light photocatalytic performance and the charge transfer process were also discussed.

\section{Experimental}

\subsection{Photocatalyst preparation}

Commercial chemicals of analytical grade and deionized water with a resistivity $>18 \mathrm{M} \Omega \bullet \mathrm{cm}-1$ were used in all the experiments. Commercial $\mathrm{ZnO}$ nanoparticles $(>99.5 \%)$ were purchased from Sigma.

$\mathrm{ZnO}$ nanosheets photocatalysts. $\mathrm{ZnO}$ nanosheet-assemblies were synthesized from the supersaturated zinc-bearing alkaline solutions, using $\mathrm{ZnO}$ and $\mathrm{NaOH}$ as the starting chemicals. $\mathrm{ZnO}$ $(1.253 \mathrm{~g})$ and $\mathrm{NaOH}(4.000 \mathrm{~g})$ were dissolved into water $(10.0 \mathrm{~mL})$ at room temperature to form a solution containing $1.54 \mathrm{~mol} \cdot \mathrm{L}^{-1} \mathrm{Zn}^{2+}$ and $10.00 \mathrm{~mol} \cdot \mathrm{L}^{-1} \mathrm{OH}$. Two $\mathrm{mL}$ of the above solution was then diluted with $98 \mathrm{ml}$ of water quickly at $60{ }^{\circ} \mathrm{C}$ to form a supersaturated system. The solution became cloudy immediately after mixing and was kept stirring at $60{ }^{\circ} \mathrm{C}$ for $1 \mathrm{~min}$ before centrifugation. The precipitates were then washed with deionized water and ethanol, and dried in an oven at $55^{\circ} \mathrm{C}$ for $24 \mathrm{~h}$. 
The thickness and BET surface area of the $\mathrm{ZnO}$ nanosheets could be tuned by pre-dissolving of different amounts of $\mathrm{Na}_{2} \mathrm{SO}_{4}$ in water. The concentration of $\mathrm{Na}_{2} \mathrm{SO}_{4}$ in the synthesis solutions was adjusted in the range of $0-1.0 \mathrm{~mol} \cdot \mathrm{L}^{-1}$.

ZnO/Ag ${ }_{3} \mathrm{PO}_{4}$ nanocomposites. The deposition of $\mathrm{Ag}_{3} \mathrm{PO}_{4}$ nanoparticles onto the $\mathrm{ZnO}$ nanosheets was carried out by an in-situ precipitation method. $\mathrm{ZnO}$ sample $(0.15 \mathrm{~g})$ was dispersed in $24 \mathrm{~mL}$ of

$0.005 \mathrm{~mol} \cdot \mathrm{L}^{-1} \mathrm{AgNO}_{3}$ solution and sonicated for $30 \mathrm{~min}$. Then $8 \mathrm{~mL}$ of $0.005 \mathrm{~mol} \cdot \mathrm{L}^{-1} \mathrm{Na}_{3} \mathrm{PO}_{4}$ solution was added drop-wise to the above solution under vigorous magnetic stirring. The resulting yellow solution was kept stirring in dark for 2 hours, then centrifuged, washed with deionized water and ethanol, followed by drying in an oven at $55{ }^{\circ} \mathrm{C}$ for $12 \mathrm{~h}$. For comparison, $\mathrm{Ag}_{3} \mathrm{PO}_{4}$ nanoparticles were prepared under the same conditions but without the addition of $\mathrm{ZnO}$.

\subsection{Characterization}

The morphology and microstructure of the samples were examined with a field emission scanning electron microscope (FESEM, JSM 7401F, JEOL, Japan) and a high-resolution transmission electron microscope (HRTEM, JEM-2010, JEOL, Japan) equipped with energy-dispersive X-ray spectroscopy. Powder X-ray diffraction (XRD) was used for phase identification using an X-ray powder diffractometer (Bruker-AXS D8 Advance, Germany) with $\mathrm{CuK} \alpha(\lambda=0.154178 \mathrm{~nm})$ radiation. The Brunauer-Emmett-Teller (BET) surface areas of the products were determined using a nitrogen adsorption analyzer (Quadrasorb-S1, Quantachrome, USA) and the pore-size distribution was estimated by the Barrett-Joyner-Halenda method. The surface electric potential was measured with the zeta potential equipment (ZETAPALS, Brookhaven Instrument Corporation). Raman spectra were recorded using He-Ne laser excitation at $532 \mathrm{~nm}$ with a Horiba Jobin Yvon LabRAM HR800 Raman 
spectrometer. Photoluminescence (PL) spectra were measured at room temperature on a Hitachi F-7000 luminescence spectrometer using a Xe lamp with an excitation wavelength of $325 \mathrm{~nm}$. Surface composition of the samples was characterized by X-ray photoelectron spectrometer (XPS, PHI-5300, PHI, USA). Electron-spin-resonance (ESR) measurements were performed on a JEOL-TE300 spectrometer operating at an X-band frequency of $9.4 \mathrm{GHz}$ and UV-visible diffuse reflectance spectra were recorded on an Agilent UV-8453 spectrophotometer.

The photo-electrochemical experiments were performed in a three-electrode quartz cells with 0.1 $\mathrm{mol} \cdot \mathrm{L}^{-1} \mathrm{Na}_{2} \mathrm{SO}_{4}$ electrolyte solution and recorded using an electrochemical system (CHI-660B, China). A xenon lamp equipped with an ultraviolet cutoff filter was employed as the visible light source $\left(\lambda>420 \mathrm{~nm}\right.$; light intensity: $\left.1 \mathrm{~mW} \cdot \mathrm{cm}^{-2}\right)$. The working electrodes were prepared by drop-casting of the photocatalyst suspensions with a concentration of $10 \mathrm{mg} \cdot \mathrm{mL}^{-1}$ onto Ti foil and drying at $55^{\circ} \mathrm{C}$ for $12 \mathrm{~h}$. Platinum wire and $\mathrm{Ag} / \mathrm{AgCl}$ were used as the counter and reference electrode, respectively.

\subsection{Photocatalytic activity measurements}

The photocatalytic activities were evaluated at $25{ }^{\circ} \mathrm{C}$ by the degradation of rhodamine $\mathrm{B}(\mathrm{RhB})$ under visible light irradiation from a $8 \mathrm{~W}$ Xe lamp equipped with an ultraviolet cutoff filter $(\lambda>420$ $\mathrm{nm})$. In a typical run, $10 \mathrm{mg}$ of the photocatalyst was dispersed in $50 \mathrm{~mL}$ of $\mathrm{RhB}$ aqueous solution (10 $\left.\mathrm{mg} \cdot \mathrm{L}^{-1}\right)$. Before irradiating, the above suspension was stirred in the dark for 30 min to achieve the adsorption-desorption equilibrium. The suspension was then illuminated under visible light, sampled at regular intervals of 10 or $15 \mathrm{~min}$ and centrifuged to remove the catalysts at $6000 \mathrm{rpm}$ for $10 \mathrm{~min}$. The UV-vis absorption spectra of the centrifuged solutions were measured by an Agilent UV-8453 spectrophotometer to determine the concentration of $\mathrm{RhB}$. The initial concentration $\left(\mathrm{C}_{0}\right)$ was 
considered to be the RhB concentration after adsorption equilibrium.

\section{Results and discussion}

\subsection{Formation of $\mathrm{ZnO}$ nanosheets with tunable BET surface areas}

In this study, $\mathrm{ZnO}$ nanosheets with tunable thickness and BET surface area were controllably synthesized by a simple solution-based self-assembly approach using $\mathrm{Na}_{2} \mathrm{SO}_{4}$ as a growth modifier. The role of $\mathrm{Na}_{2} \mathrm{SO}_{4}$ concentration on the morphology of $\mathrm{ZnO}$ nanosheets was firstly examined by SEM and TEM. Fig. S1 and Fig. 1a-d show the low- and high-magnification SEM images, respectively of the $\mathrm{ZnO}$ samples formed in the presence of $0-1.0 \mathrm{~mol} \cdot \mathrm{L}^{-1} \mathrm{Na}_{2} \mathrm{SO}_{4}$. The SEM results demonstrate that the obtained $\mathrm{ZnO}$ displays a hierarchical porous network structure composed of intersecting nanosheets. With the increase of $\mathrm{Na}_{2} \mathrm{SO}_{4}$ concentration from 0 to $1.0 \mathrm{~mol} \cdot \mathrm{L}^{-1}$, the size of the nanosheet-assemblies decreased from 5-10 $\mu \mathrm{m}$ to $0.5-1 \mu \mathrm{m}$ and the thickness of nanosheets gradually decreased from $30-50 \mathrm{~nm}$ to $10-15 \mathrm{~nm}$. Some of the nanosheets formed at $\left[\mathrm{Na}_{2} \mathrm{SO}_{4}\right]=1.0$ mol $\cdot \mathrm{L}^{-1}$ became semi-transparent due to their ultrathin nature. The density of the assembled nanosheets also decreased with increasing $\mathrm{Na}_{2} \mathrm{SO}_{4}$ concentration. At $\left[\mathrm{Na}_{2} \mathrm{SO}_{4}\right]=1.0 \mathrm{~mol} \cdot \mathrm{L}^{-1}$, the nanosheets were loosely assembled or even dis-assembled. The TEM observation (Fig. 1e and f) confirmed that the thickness of nanosheets decreased from 30-50 nm to $10-15 \mathrm{~nm}$ with increasing $\mathrm{Na}_{2} \mathrm{SO}_{4}$ concentration from 0 to $1.0 \mathrm{~mol} \cdot \mathrm{L}^{-1}$. The HRTEM images along with the Fast Fourier Transform (FFT) pattern (Fig. S2) revealed the preferential growth of the nanosheets along [001], with (100) planes as exposed surfaces. The XRD patterns (Fig. S3a) of all the four samples can be indexed to würtzite $\mathrm{ZnO}$ (JCPDS 36-1451). The peak broadening and decreasing intensity of the (100) diffraction peaks (inset in Fig. S3b) indicated a decrease of the (100) crystallite size and poor 
crystallization of $\mathrm{ZnO}$. The relative intensity ratio of (100) to (002) $\left(\mathrm{I}_{(100)} / \mathrm{I}_{(002)}\right)$ gradually decreased from 1.81 to 0.55 with increasing $\mathrm{Na}_{2} \mathrm{SO}_{4}$ concentration from 0 to $1.0 \mathrm{~mol} \cdot \mathrm{L}^{-1}$, which implies that the growth of $\mathrm{ZnO}$ in (100) orientation was inhibited in the presence of $\mathrm{Na}_{2} \mathrm{SO}_{4}$. These results are consistent with SEM and TEM observations, which showed a decreasing thickness of nanosheets with increasing $\mathrm{Na}_{2} \mathrm{SO}_{4}$ concentration.

The variation in nanosheets' size, thickness and density resulted in a variation of their BET surface areas and pore structures (Figs. $2 \mathrm{a}$ and $\mathrm{b}$ ). The BET surface areas increased with increasing $\mathrm{Na}_{2} \mathrm{SO}_{4}$ concentration, i.e., the surface areas were $6.7,18.1,27.3$ and $34.5 \mathrm{~m}^{2} / \mathrm{g}$ at $\mathrm{Na}_{2} \mathrm{SO}_{4}$ concentrations of $0,0.02,0.1$ and $1.0 \mathrm{~mol} \cdot \mathrm{L}^{-1}$, respectively. The BET surface area of the nanosheets obtained here in $1.0 \mathrm{~mol} \cdot \mathrm{L}^{-1} \mathrm{Na}_{2} \mathrm{SO}_{4}$ was higher than many of those reported previously for $\mathrm{ZnO}$ nanosheets or even porous nanosheets [22-24]. The pore size distribution curves (Fig. 2b) revealed that the volume of the mesopores increased with increasing $\mathrm{Na}_{2} \mathrm{SO}_{4}$ concentration. In addition, the average size of the mesopores increased from ca. $15 \mathrm{~nm}$ to $30 \mathrm{~nm}$, which is consistent with the SEM observation that showed the nanosheets being loosely assembled in the presence of concentrated $\mathrm{Na}_{2} \mathrm{SO}_{4}$. The $\mathrm{ZnO}$ nanosheet-assemblies with higher surface area and more open pore structures may also find applications as adsorbents or catalyst supports in addition to photocatalysts. All the above results indicated that the growth and self-assembly of the $\mathrm{ZnO}$ nanosheets could be affected by $\mathrm{Na}_{2} \mathrm{SO}_{4}$ addition under the present synthesis conditions. 

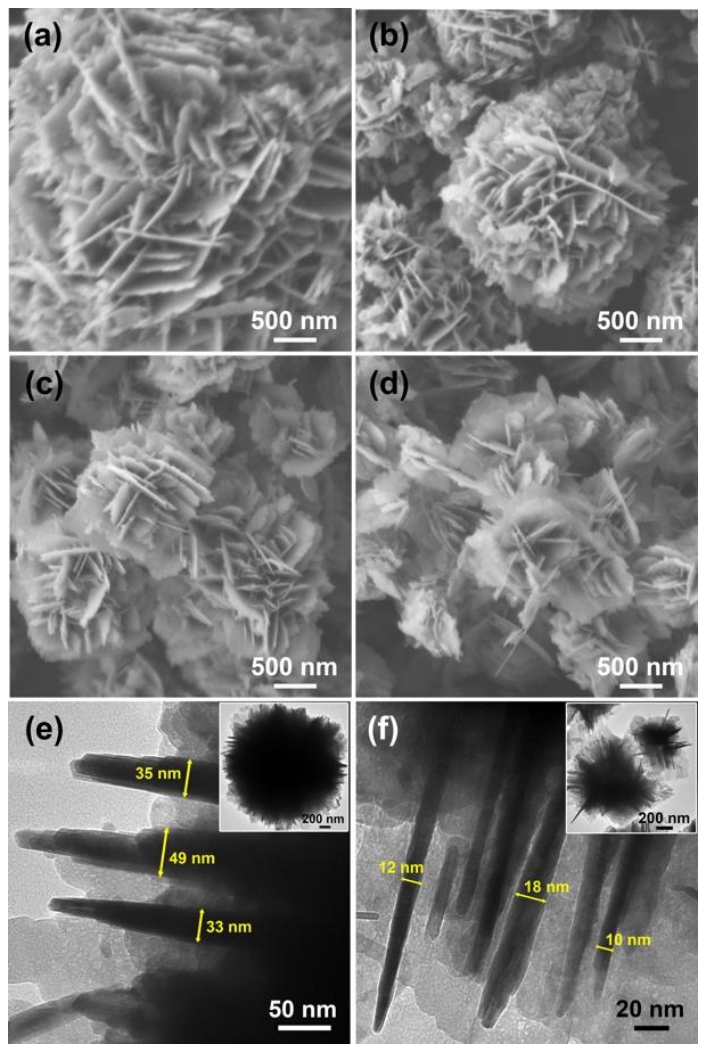

Fig.1. SEM images (a-d) and TEM images (e, f) of the $\mathrm{ZnO}$ nanosheets formed in the presence of different $\mathrm{Na}_{2} \mathrm{SO}_{4}$ concentrations $\left(\mathrm{mol} \cdot \mathrm{L}^{-1}\right)$ : (a, e) 0 ; (b) 0.02 ; (c) 0.1 ; (d, f) 1.0 .
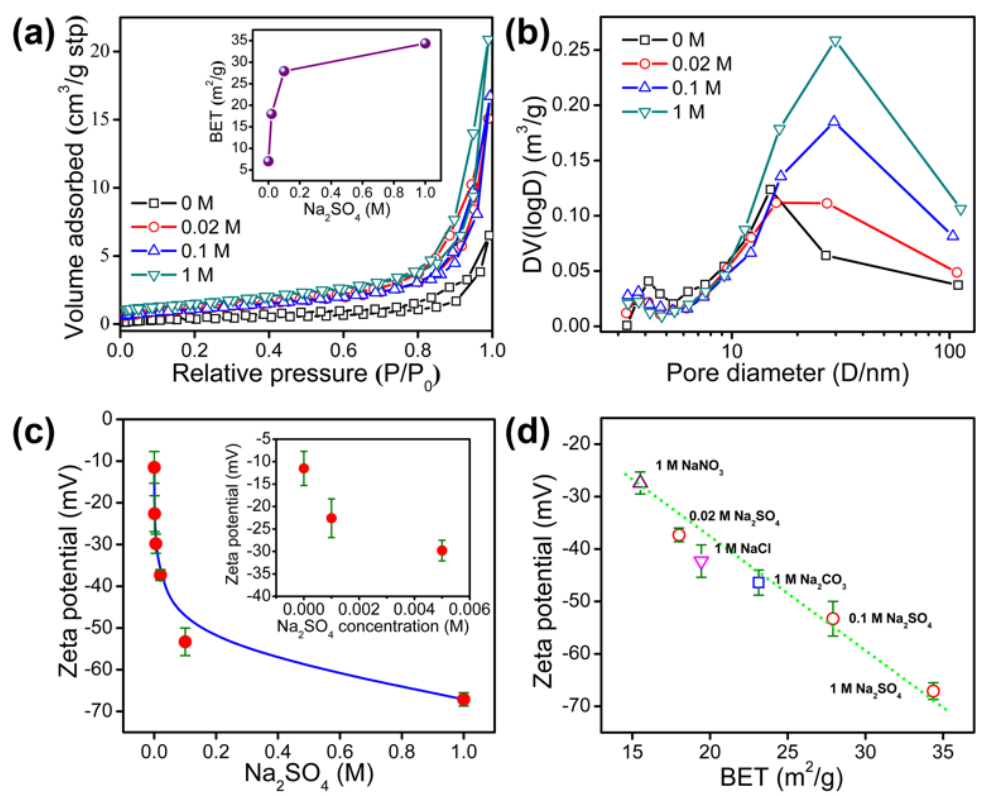

Fig. 2. $\mathrm{N}_{2}$ adsorption-desorption isotherms (a), pore size distributions (b) of the $\mathrm{ZnO}$ nanosheets 
formed in the presence of $\mathrm{Na}_{2} \mathrm{SO}_{4}$ with different concentrations, inset in Figure 2a is the variation of specific surface area of the samples with $\mathrm{Na}_{2} \mathrm{SO}_{4}$ concentration; (c) variation of zeta potential of the $\mathrm{ZnO}$ nanosheets with $\mathrm{Na}_{2} \mathrm{SO}_{4}$ concentration; (d) summarized zeta potentials and specific surface areas of the samples formed under different conditions (the dashed line serves as a guide line).

The effects of salt addition can be explained by an electrostatics-controlled growth and self-assembly mechanism. Insight into the time-dependent growth process of the $\mathrm{ZnO}$ nanosheets in the absence of $\mathrm{Na}_{2} \mathrm{SO}_{4}$ revealed that the nanosheets were formed by an ultra-rapid oriented attachment and self-assembly process, as revealed in Fig. S4. ZnO nanocrystals were immediately formed after dilution due to the high supersaturation (Fig. S4a), subsequently assembled into nanosheets by the two-dimensional oriented attachment process (Fig. S4b) with many nanocrystal-boundaries still remaining in the nanosheets (Fig. S4c), and further assembled into well-defined nanosheet-assemblies (Fig. S4d). The whole self-assembly process was superfast and could be completed within only 1 minute. The driving forces that assemble nanocrystals into nanosheets were generally attributed to anisotropic hydrophobic attraction or electrostatic interactions derived from dipole moments and surface charges $[25,26]$. In the present case, hydrophobic attraction could be ruled out since no organic template/surfactant was added. Therefore, the surface charge of the nanocrystals may affect the oriented attachment process. Fig. $2 \mathrm{c}$ shows the variation of zeta potential of the $\mathrm{ZnO}$ nanosheets with $\mathrm{Na}_{2} \mathrm{SO}_{4}$ concentration. The zeta potential of the $\mathrm{ZnO}$ nanosheets dropped from $-12.1 \mathrm{eV}$ to -67.5 $\mathrm{eV}$ with increasing $\mathrm{Na}_{2} \mathrm{SO}_{4}$ concentration from 0 to $1.0 \mathrm{~mol} \cdot \mathrm{L}^{-1}$. The zeta potentials indicate that $\mathrm{ZnO}$ became more negatively charged in the presence of $\mathrm{Na}_{2} \mathrm{SO}_{4}$, thus the attachment and self-assembly of the initial nanocrystals were hindered due to the electrostatic repulsion, which led to the formation of 
thinner and smaller nanosheets with looser packing. Such effects could also be observed in the presence of other salts such as $\mathrm{NaNO}_{3}, \mathrm{Na}_{2} \mathrm{CO}_{3}$ and $\mathrm{NaCl}$, as displayed in the SEM images in Fig. S5. The summarized data of zeta potentials and BET surface areas of the $\mathrm{ZnO}$ nanosheets under different conditions are shown in Fig. 2d. The clear correlation between BET surface areas and surface charges suggested that the growth and self-assembly of $\mathrm{ZnO}$ nanosheets were controlled by electrostatic process. The effects of salts addition on the attachment behavior of $\mathrm{ZnO}$ nanocrystals may be explained by Derjaguin Landau Verwey Overbeek (DLVO) theory, in which the main force between the nanocrystals were electrical double layer repulsion derived from the ion adsorption layers around the nanocrystals [27]. In the present case, the effects of $\mathrm{Na}_{2} \mathrm{SO}_{4}$ addition was more significant compared to other salts, which could be attributed to the preferential adsorption of $\mathrm{SO}_{4}{ }^{2-}$ onto the (100) planes of $\mathrm{ZnO}$, as revealed in our previous work [28]. Such electrostatic-controlled synthesis strategy has also been employed in the hydrothermal growth of $\mathrm{ZnO}$ nanowires with tunable aspect ratios [29]. However, the electrostatic-mediated self-assembly of $\mathrm{ZnO}$ nanocrystals into three-dimensional hierarchical structures in a controlled manner has not been reported yet. The presently developed method may provide an alternative route to control the BET surface areas of solution-processed inorganic nanostructures. Moreover, the adsorbed inorganic ions in the current work (see EDS spectra in Fig. S6) could be completely removed by washing unlike many organic surfactants used in other works as growth modifiers [30,31], which may inhibit the active sites of the photocatalysts.

\subsection{Identification of oxygen defects in $\mathrm{ZnO}$ nanosheets}

As shown above, $\mathrm{ZnO}$ nanosheets were formed from highly-supersaturated solution by an ultra-rapid self-assembly process [32]. Such a non-equilibrium synthesis process was generally 
considered to lead to the generation of a high-concentration of defects [33-35]. Multiple techniques were employed to identify the defect states of the $\mathrm{ZnO}$ nanosheets with different BET surface areas formed in 0 and $1 \mathrm{~mol} \cdot \mathrm{L}^{-1} \mathrm{Na}_{2} \mathrm{SO}_{4}$ solutions, which were denoted as ZNS-1 and ZNS-4, respectively. Commercial $\mathrm{ZnO}$ nanoparticles with an average diameter of $20 \mathrm{~nm}$ and a BET surface area of 43.4 $\mathrm{m}^{2} / \mathrm{g}$ (denoted as ZnO NPs) were used as a reference (Fig. S7). Fig. 3a shows the Raman spectra of the different samples. Little difference was observed among the peaks located at $333 \mathrm{~cm}^{-1}, 380 \mathrm{~cm}^{-1}$ and $439 \mathrm{~cm}^{-1}$, which corresponded to $2 \mathrm{E}_{2}$ (low), $\mathrm{A}_{1}(\mathrm{TO})$ and $\mathrm{E}_{2}$ (high) modes of $\mathrm{ZnO}$, respectively. The $\mathrm{E}_{1}(\mathrm{LO})$ modes (located at $582 \mathrm{~cm}^{-1}$ ) of the samples emerged as a red shift compared with that of the bulk $\mathrm{ZnO}\left(591 \mathrm{~cm}^{-1}\right)$, which may be assigned to the oxygen deficiencies such as oxygen vacancies $\left(\mathrm{V}_{\mathrm{O}}\right)$ in $\mathrm{ZnO}$ [36]. The order of the intensities of $\mathrm{E}_{1}(\mathrm{LO})$ mode was ZNS-4>ZNS-1>>ZnO NPs (inset in Fig. 3a), indicating the much higher concentration of oxygen-related defects in $\mathrm{ZnO}$ nanosheets compared to $\mathrm{ZnO}$ nanoparticles. Fig. $3 \mathrm{~b}$ shows the room temperature PL spectra of the samples. The ZnO NPs displayed an ultraviolet (UV) emission peak with maximal intensity at around $384 \mathrm{~nm}$, which was due to the excitonic recombination of the photogenerated holes in the valence band (VB) and the electrons in the conduction band (CB) [37]. However, the characteristic UV emission was not clearly distinguished in the as-prepared nanosheets, indicating that high concentrations of defects were introduced into the samples. The multi-peak bands of the nanosheets in the range of 400-480 nm were generally ascribed to the recombination of the electrons from the shallow level donor of zinc interstitials $\left(\mathrm{Zn}_{\mathrm{i}}\right)$ and a series of extended $\mathrm{Zn}_{\mathrm{i}}$ states to holes in the valence band. In the green-yellow region, a very weak green emission centered at about $500 \mathrm{~nm}$ was observed for $\mathrm{ZnO} \mathrm{NPs}$, while the nanosheets exhibited intensive green-yellow emission centered at $550 \mathrm{~nm}$, which could be divided into 
two peaks, including the green emission at $\sim 520 \mathrm{~nm}$ originated from singly charged oxygen vacancies $\left(\mathrm{V}_{\mathrm{O}} \bullet\right)$ and the yellow one at $\sim 580 \mathrm{~nm}$ originated from doubly charged oxygen vacancies $\left(\mathrm{V}_{\mathrm{O}}^{\bullet \bullet}\right)[35$, 38].
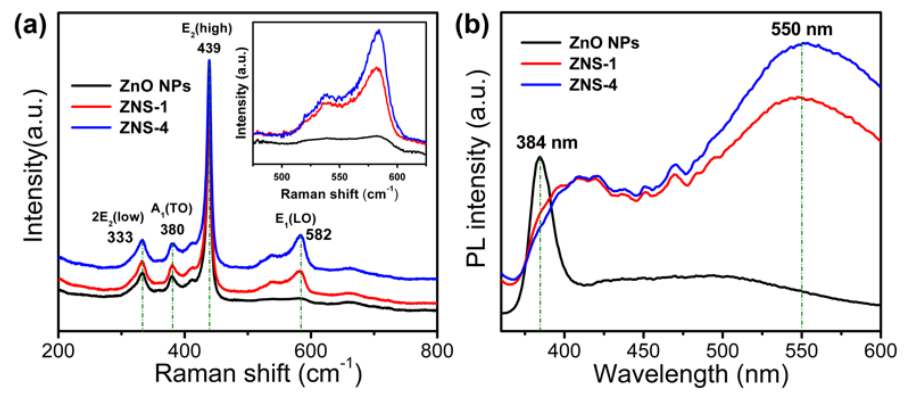

Fig. 3. Raman spectra (a) and room-temperature PL spectra (b) of ZNS-1, ZNS-4 and ZnO nanoparticles. Inset in Figure $3 \mathrm{a}$ is the enlarged $\mathrm{E}_{1}(\mathrm{LO})$ modes.

Since Raman and PL spectra give information on both bulk and surface oxygen defects, XPS and ESR spectra were employed to further characterize the surface properties of the $\mathrm{ZnO}$ samples. Fig. $4 \mathrm{a}$ shows the high-resolution O1s spectra of the three samples. Two species centered at the banding energies of ca. 530.2 530.3 eV and 531.6 531.7 eV were denoted as $\mathrm{O} 1$ and $\mathrm{O} 2$, respectively. The species of $\mathrm{O} 1$ were originated from the lattice oxygen anions $\left(\mathrm{O}^{2-}\right)$ in würtzite structure, while the species of $\mathrm{O} 2$ belonged to the $\mathrm{O}_{\mathrm{x}}{ }^{-}$ions $\left(\mathrm{O}^{-}\right.$and $\left.\mathrm{O}_{2}{ }^{-}\right)$in the oxygen-deficient regions caused by $\mathrm{V}_{\mathrm{O}}$ on $\mathrm{ZnO}$ surface [39]. The ratios of O2 species on the surface of ZnO NPs, ZNS-1 and ZNS-4 were 13.9, 46.8 and $54.1 \%$, respectively, indicating that the as-prepared $\mathrm{ZnO}$ nanosheets had a high concentration of surface oxygen vacancies. Fig. $4 \mathrm{~b}$ shows the ESR spectra of the three samples. The signal with $\mathrm{g}$-factor $=2.01$ is close to the free-electron value ( $\mathrm{g} 2.0023$ ), which was previously attributed to an unpaired electron trapped on a surface oxygen vacancy site [40]. This is because the surface oxygen vacancy is prone to adsorb atmospheric $\mathrm{O}_{2}$ molecules and form $\cdot \mathrm{O}_{2}^{-}$to generate such a signal [41]. 
The nanosheets had a much higher ESR signal at $\mathrm{g}=2.01$ in contrast to $\mathrm{ZnO}$ NPs. All the results discussed above confirmed the existence of a high concentration of oxygen vacancies in the as-prepared $\mathrm{ZnO}$ nanosheets. Moreover, a higher concentration of surface oxygen vacancies was observed for ZNS-4 compared with ZNS-1 owing to its higher surface to volume ratio, suggesting that increasing the BET surface area of $\mathrm{ZnO}$ was an effective method to enrich its surface oxygen vacancies, which are useful to the visible-light photocatalytic process.
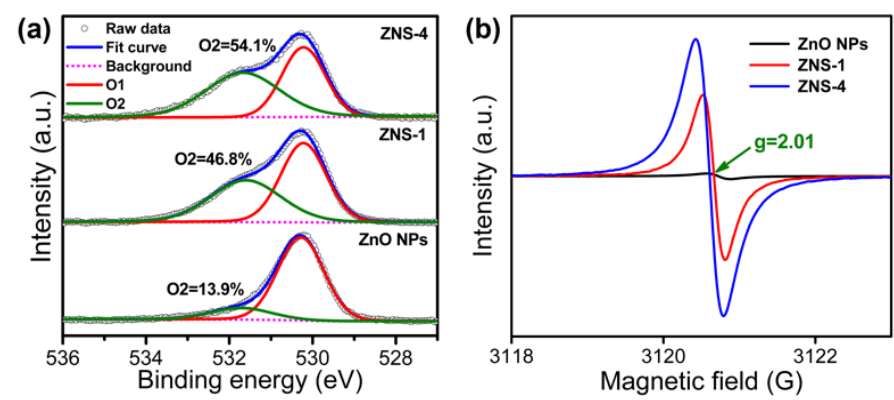

Fig. 4. High-resolution O1s XPS spectra (a) and ESR spectra (b) of ZNS-1, ZNS-4 and ZnO NPs.

The optical properties of ZNS-1, ZNS-4 and ZnO NPs were examined by UV-visible diffuse reflectance spectroscopy to evaluate the effect of oxygen vacancy on the absorption characteristics, as shown in Fig. 5a. ZnO NPs only absorbed the light with $\lambda<420 \mathrm{~nm}$ owing to its intrinsic wide band gap, while the absorption curves of as-prepared $\mathrm{ZnO}$ nanosheets exhibited a red shift to longer wavelength compared to the ZnO NPs reference while the ZNS-4 sample showed the highest absorption in visible-light region $(\lambda>420 \mathrm{~nm})$. The large absorption tail occurring in the visible-light regions of the $\mathrm{ZnO}$ nanosheets was attributed to the rich oxygen vacancies, which generated a defect-isolated state of about $0.8 \mathrm{eV}$ below the conduction band of $\mathrm{ZnO}$, promoting the visible-light absorption [42, 43]. Therefore, we can conclude that the visible-light absorption of $\mathrm{ZnO}$ could be effectively improved by introducing oxygen vacancies, and the absorption capacity was related to the 
concentration of oxygen vacancies.
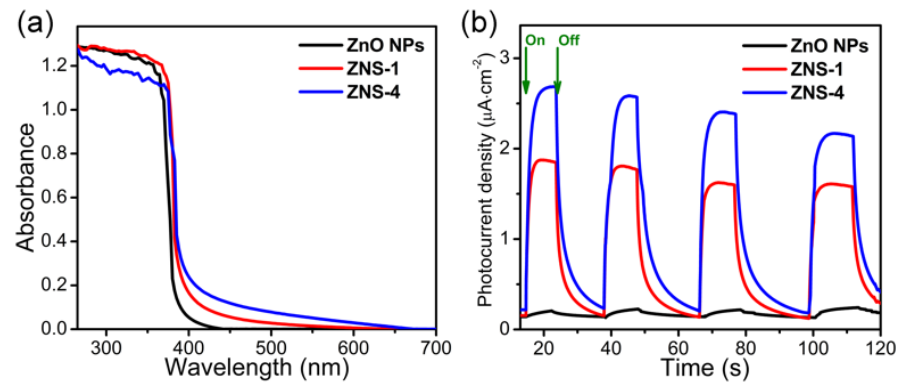

Fig. 5. (a) UV-Vis diffuse reflectance spectra of of ZNS-1, ZNS-4 and ZnO NPs; (b) photocurrent response of the ZNS-1, ZNS-4 and ZnO NPs electrodes under the irradiation of visible-light $(\lambda>420$ $\mathrm{nm})$.

The photocurrent responses of the samples were tested on a photoelectrochemical test device to further investigate the activity of $\mathrm{ZnO}$ in visible-light region. Fig. 5b shows the photocurrents responses of ZNS-1, ZNS-4 and ZnO NPs as a function of time with visible light-on and -off cycles. $\mathrm{ZnO}$ NPs electrode showed very weak photocurrent response under visible-light irradiation $(\lambda>420$ $\mathrm{nm}$ ), while the as-prepared ZNS-1 and ZNS-4 samples of ZnO nanosheets showed much larger photocurrent responses, which are about 9 and 13 times higher, respectively than that of $\mathrm{ZnO}$ NPs (Fig. 5b). This suggests that ZNS-1 and ZNS-4 samples are expected to have enhanced generation and separation efficiency of photogenerated carries under visible-light. The highest photocurrent of ZNS-4 could be attributed to the highest concentration of surface oxygen vacancies derived from the increased surface to volume ratio, which may lead to the improved visible-light absorption and surface oxygen-vacancy-mediated electrons/holes generation and separation [16].

\subsection{Photocatalytic performance and mechanisms}

The photocatalytic performances of ZNS-1, ZNS-4 and ZnO NPs were evaluated by degradation 
of $\mathrm{RhB}$ under visible-light irradiation $(\lambda>420 \mathrm{~nm})$. As shown in Fig. 6a, the $\mathrm{ZnO}$ NPs reference shows very low activity in photodegradation due to its poor visible-light absorption capacity, and only about $9.4 \%$ of $\mathrm{RhB}$ was degraded after 90 min of irradiation, even though it has a larger BET surface area $\left(43.4 \mathrm{~m}^{2} / \mathrm{g}\right)$. In contrast, the degradation ratio of $\mathrm{RhB}$ was 35.3 and $55.1 \%$ for ZNS-1 and ZNS-4 after $90 \mathrm{~min}$, respectively. The enhanced visible-light photocatalytic activity could be explained by the oxygen vacancy-enhanced visible-light absorption and charge separation. When defect-rich $\mathrm{ZnO}$ nanosheets were under visible-light illumination, the electrons in the VB could be excited to the oxygen vacancy induced defect energy level due to the lowered energy difference $(\Delta \mathrm{E}=2.4 \mathrm{eV})$. The holes left behind in the VB can react with water adhering to the surfaces of $\mathrm{ZnO}$ to form hydroxyl radicals $(\cdot \mathrm{OH})$ which can result in the oxidation of the organic dye, while the photogenerated electrons could get trapped in the surface oxygen defects working as electron acceptors, leading to the occurrence of the redox reactions [19]. Therefore, the surface oxygen defects not only improve the visible-light absorption of $\mathrm{ZnO}$, but also act as the active sites for photocatalytic reactions, which facilitated the $\mathrm{e}^{-} / \mathrm{h}^{+}$separation and prevented their recombination. Therefore, the ZNS-4 sample with highest concentration of surface oxygen vacancies exhibited significantly enhanced visible-light photocatalytic activity, as expected. 

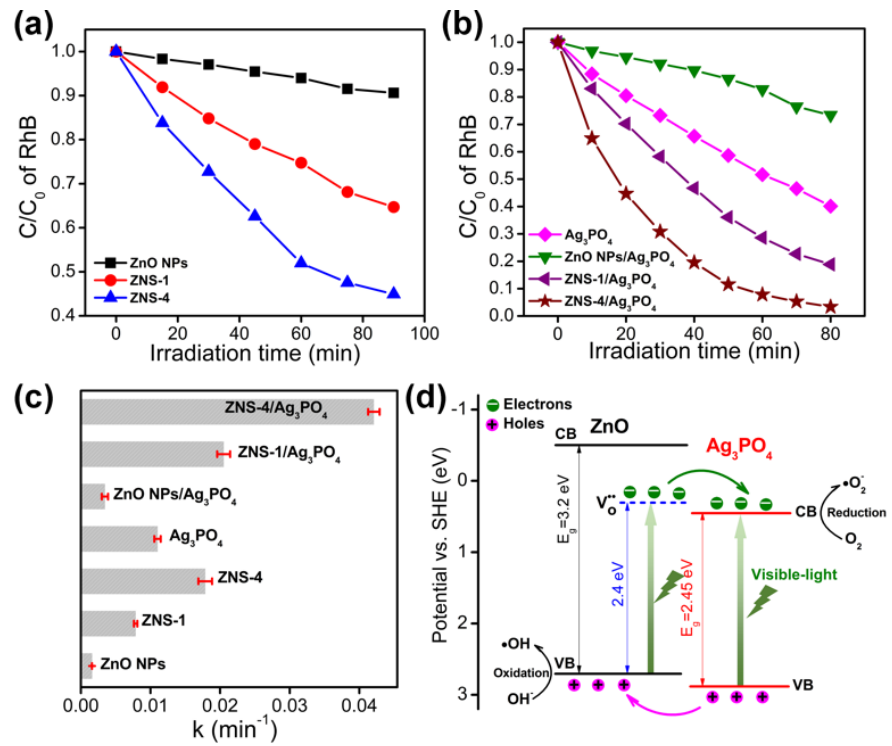

Fig. 6. (a, b) Photocatalytic degradation of RhB under visible-light irradiation in the presence of different $\mathrm{ZnO}$ or $\mathrm{ZnO} / \mathrm{Ag}_{3} \mathrm{PO}_{4}$ nanocomposite samples; (c) apparent rate constants $\left(\mathrm{k}, \mathrm{min}^{-1}\right)$ of the tested samples determined by the pseudo first-order kinetic equation; (d) schematic illustration of the band structure and charge-transfer process of oxygen defect-rich $\mathrm{ZnO}$ coupled with $\mathrm{Ag}_{3} \mathrm{PO}_{4}$ under visible-light illumination.

Sheet-like structures with high surface areas can easily combine with other functional particles through the two faces of the sheets, which is another approach to further enhance the visible-light photocatalytic activity [23]. Although the activation of $\mathrm{ZnO}$ under visible-light irradiation by hybridization with various materials including noble metal nanoparticles, narrow band-gap semiconductors and carbon nanometerials [44-46], have been widely reported, there were only a few reports related to the synergistic effect of surface oxygen defects of $\mathrm{ZnO}$ by hybridizing with a second phase. For example, enhanced photocatalytic activities were achieved by hybridization of oxygen defect-rich $\mathrm{ZnO}$ nanostructure with Ag nanoparticles [21] or graphene nanosheets [16]. However, such synergistic effect has not been reported yet for defect-rich $\mathrm{ZnO}$ coupled with other narrow band-gap 
semiconductors. Herein, therefore, we also investigated the synergistic effect of surface oxygen defects by combining $\mathrm{Ag}_{3} \mathrm{PO}_{4}$ as a second phase, i.e., $\mathrm{ZnO} / \mathrm{Ag}_{3} \mathrm{PO}_{4}$ nanocomposites as a case study. $\mathrm{Ag}_{3} \mathrm{PO}_{4}$ nanoparticles were deposited onto the surfaces of $\mathrm{ZnO}$ nanosheets by a simple in-situ precipitation method. Representative XRD pattern of the as-obtained ZNS-4/Ag ${ }_{3} \mathrm{PO}_{4}$ nanocomposite confirmed the coexistence of $\mathrm{ZnO}$ and $\mathrm{Ag}_{3} \mathrm{PO}_{4}$ phases (Fig. S8a). The TEM and HRTEM images (Figs. S8b and c) revealed that $\mathrm{Ag}_{3} \mathrm{PO}_{4}$ nanoparticles with diameters of 5-30 nm were coated onto the surfaces of $\mathrm{ZnO}$ nanosheets. UV-vis spectrum (Fig. S8d) indicated that the absorption in the visible-light region of the $\mathrm{ZNS}-4 / \mathrm{Ag}_{3} \mathrm{PO}_{4}$ nanocomposite increased significantly due to the narrow band gap of $\mathrm{Ag}_{3} \mathrm{PO}_{4}(2.45 \mathrm{eV})$, which can absorb light with a wavelength of less than $503 \mathrm{~nm}$ [47]. The increased visible-light absorption of the nanocomposites led to the enhancement of the visible-light photocatalytic activities, as shown in Fig. 6b. The apparent rate constants of the tested samples were determined by the pseudo first-order kinetic equation (Fig. S9) and displayed in Fig. 6c and these results clearly demonstrate the photocatalytic activity order of the samples. For ZNS- $1 / \mathrm{Ag}_{3} \mathrm{PO}_{4}$ and $\mathrm{ZNS}-4 / \mathrm{Ag}_{3} \mathrm{PO}_{4}$ samples, the photodegradation ratio of $\mathrm{RhB}$ increased to 81.1 and $96.6 \%$ after $80 \mathrm{~min}$ of irradiation. The determined rate constants for the above two samples were 0.0205 and $0.0421 \mathrm{~min}^{-1}$, respectively, which are higher than that of either $\mathrm{ZnO}$ nanosheets or $\mathrm{Ag}_{3} \mathrm{PO}_{4}$ samples, indicating the enhanced visible-light photocatalytic activity of the $\mathrm{ZnO} / \mathrm{Ag}_{3} \mathrm{PO}_{4}$ nanocomposites. However, the $\mathrm{ZnO} \mathrm{NPs} / \mathrm{Ag}_{3} \mathrm{PO}_{4}$ sample showed much lower activity compared to solely $\mathrm{Ag}_{3} \mathrm{PO}_{4}$ even though the total weight of the photocatalyst was kept constant $(10 \mathrm{mg})$, due to the low loading amount of $\mathrm{Ag}_{3} \mathrm{PO}_{4}(\sim 10.0 \mathrm{wt} \%)$ on $\mathrm{ZnO}$ NPs with few defects, which are not active in visible light. These results implied that surface oxygen defects of $\mathrm{ZnO}$ played a critical role of in the 
photocatalytic performance of the nanocomposites. Therefore, the enhanced visible-light photocatalytic activity of $\mathrm{ZnO}$ nanosheets/ $\mathrm{Ag}_{3} \mathrm{PO}_{4}$ nanocomposites could be attributed to not only the increased visible-light absorption, but also the synergistic effect of surface oxygen vacancies and $\mathrm{Ag}_{3} \mathrm{PO}_{4}$ coupling. The VB and $\mathrm{CB}$ positions of $\mathrm{Ag}_{3} \mathrm{PO}_{4}$ vs. NHE (normal hydrogen electrode) are 2.9 and $0.45 \mathrm{eV}$ [48], lower than the VB $(2.6 \mathrm{eV})$ and oxygen defects energy level $(0.2 \mathrm{eV})$ of $\mathrm{ZnO}$, respectively, thus an energy level matching is established between $\mathrm{Ag}_{3} \mathrm{PO}_{4}$ and $\mathrm{ZnO}$ with rich oxygen-vacancies, as illustrated in Fig. 6d. Under visible-light illumination, the electrons in VB of $\mathrm{ZnO}$ and $\mathrm{Ag}_{3} \mathrm{PO}_{4}$ could be excited to the oxygen-vacancy defect energy level of $\mathrm{ZnO}$ and $\mathrm{CB}$ of $\mathrm{Ag}_{3} \mathrm{PO}_{4}$, respectively, thus leading to highly increased visible-light absorption. On the other hand, the excited electrons in the defect energy level of $\mathrm{ZnO}$ would transfer to the $\mathrm{CB}$ of $\mathrm{Ag}_{3} \mathrm{PO}_{4}$, and the holes in the VB of $\mathrm{Ag}_{3} \mathrm{PO}_{4}$ would transfer to the VB of the $\mathrm{ZnO}$. Such spatial separation of the photogenerated electrons/holes within the nanocomposite by a direct Z-scheme mechanism significantly reduced the electron-hole recombination ratio, which promoted the subsequent redox reactions leading to a much higher visible-light photocatalytic performance of the nanocomposites [49, 50]. These results suggest that $\mathrm{ZnO}$ nanostructures with rich oxygen vacancies are more suitable for constructing functional nanocomposites for visible-light photocatalysis applications.

\section{Conclusion}

In summary, $\mathrm{ZnO}$ nanosheets with high surface area and rich oxygen vacancies were synthesized via a salt-assisted ultrarapid solution method. An electrostatic-controlled self-assembly mechanism was proposed for the effects of salt addition. The results obtained by Raman, PL, XPS and ESR analyses indicated that the as-prepared nanosheets had much higher concentration of oxygen 
vacancies compared to $\mathrm{ZnO}$ nanoparticles. The increased BET surface areas achieved here contributed to the increase of surface oxygen vacancies, which could improve the visible-light absorption and act as active sites for photocatalytic reactions, leading to the enhancement of photocurrent and photocatalytic activities of the $\mathrm{ZnO}$ nanosheets under visible-light illumination. Hybridization of $\mathrm{ZnO}$ nanosheets with $\mathrm{Ag}_{3} \mathrm{PO}_{4}$ nanoparticles further improved the visible-light photocatalytic activity, which was attributed to the efficient charge transfer between $\mathrm{ZnO}$ and $\mathrm{Ag}_{3} \mathrm{PO}_{4}$ through energy level matching, suggesting a synergistic effect of surface oxygen vacancies and $\mathrm{Ag}_{3} \mathrm{PO}_{4}$ coupling.

\section{Acknowledgements}

This work was financially supported by the National Science Foundation of China (Nos. 51174125, 51234003 and 51374138), National Key Technology Research and Development Program of China (2013BAC14B02). Jing Wang is grateful for the scholarship from China Scholarship Council (No. 201506210232).

\section{References}

[1] S. Sakthivel, B. Neppolian, M. Shankar, B. Arabindoo, M. Palanichamy, V. Murugesan, Sol. Energy Mater. Sol. Cells, 77 (2003) 65-82.

[2] T. Bak, J. Nowotny, M. Rekas, C. Sorrell, Int. J. Hydrogen Energy, 27 (2002) 991-1022.

[3] M. Miyauchi, A. Nakajima, T. Watanabe, K. Hashimoto, Chem. Mater., 14 (2002) 2812-2816.

[4] J. C. Sin, S. M. Lam, I. Satoshi, K. T. Lee, A.R. Mohamed, Appl. Catal. B: Environ., 148 (2014) 258-268.

[5] Y. Lu, Y. Lin, D. Wang, L. Wang, T. Xie, T. Jiang, Nano Res., 4 (2011) 1144-1152. 
[6] S. Liu, C. Li, J. Yu, Q. Xiang, CrystEngComm, 13 (2011) 2533-2541.

[7] S. Rehman, R. Ullah, A. Butt, N. Gohar, J. Hazard. Mater., 170 (2009) 560-569.

[8] J. Wang, Z. Wang, B. Huang, Y. Ma, Y. Liu, X. Qin, X. Zhang, Y. Dai, ACS Appl. Mat. Interfaces, 4 (2012) 4024-4030.

[9] X. Liu, X. Wu, H. Cao, R. Chang, J. Appl. Phys., 95 (2004) 3141-3147.

[10] F. Liu, Y.H. Leung, A.B. Djurišić, A.M.C. Ng, W.K. Chan, K.L. Ng, K.S. Wong, C. Liao, K. Shih, C. Surya, J. Phys. Chem. C, 118 (2014) 22760-22767.

[11] K. Tam, C. Cheung, Y. Leung, A.B. Djurišić, C. Ling, C. Beling, S. Fung, W. Kwok, W. Chan, D. Phillips, J. Phys. Chem. B, 110 (2006) 20865-20871.

[12] S. Mukhopadhyay, P.P. Das, S. Maity, P. Ghosh, P.S. Devi, Appl. Catal. B: Environ., 165 (2015) $128-138$.

[13] H. L. Guo, Q. Zhu, X. L. Wu, Y. F. Jiang, X. Xie, A. W. Xu, Nanoscale, 7 (2015) 7216-7223.

[14] E. Grabowska, J.W. Sobczak, M. Gazda, A. Zaleska, Appl. Catal. B: Environ., 117 (2012) 351-359.

[15] X. Chen, L. Liu, Y.Y. Peter, S.S. Mao, Science, 331 (2011) 746-750.

[16] X. Bai, L. Wang, R. Zong, Y. Lv, Y. Sun, Y. Zhu, Langmuir, 29 (2013) 3097-3105.

[17] M. Kong, Y. Li, X. Chen, T. Tian, P. Fang, F. Zheng, X. Zhao, J. Am. Chem. Soc., 133 (2011) 16414-16417.

[18] M.Y. Guo, A.M.C. Ng, F. Liu, A.B. Djurišić, W.K. Chan, H. Su, K.S. Wong, J. Phys. Chem. C, 115 (2011) 11095-11101.

[19] J. Wang, P. Liu, X. Fu, Z. Li, W. Han, X. Wang, Langmuir, 25 (2008) 1218-1223. 
[20] G. Li, T. Hu, G. Pan, T. Yan, X. Gao, H. Zhu, J. Phys. Chem. C, 112 (2008) 11859-11864.

[21] Y. Zheng, L. Zheng, Y. Zhan, X. Lin, Q. Zheng, K. Wei, Inorg. Chem., 46 (2007) 6980-6986.

[22] G.-X. Tong, F. F. Du, Y. Liang, Q. Hu, R. N. Wu, J. G. Guan, X. Hu, J. Mater. Chem. B, 1 (2013)

454-463.

[23] Y. Hong, C. Tian, B. Jiang, A. Wu, Q. Zhang, G. Tian, H. Fu, J. Mater. Chem. A, 1 (2013) $5700-5708$.

[24] H. Lu, S. Wang, L. Zhao, J. Li, B. Dong, Z. Xu, J. Mater. Chem., 21 (2011) 4228-4234.

[25] Z. Zhang, Z. Tang, N.A. Kotov, S.C. Glotzer, Nano lett., 7 (2007) 1670-1675.

[26] Z. Tang, Z. Zhang, Y. Wang, S.C. Glotzer, N.A. Kotov, Science, 314 (2006) 274-278.

[27] S. W. Bian, I.A. Mudunkotuwa, T. Rupasinghe, V.H. Grassian, Langmuir, 27 (2011) 6059-6068.

[28] L. Yang, J. Wang, L. Xiang, Particuology, 19 (2015) 113-117.

[29] J. Joo, B.Y. Chow, M. Prakash, E.S. Boyden, J.M. Jacobson, Nat. Mater. 10 (2011) 596-601.

[30] E.O. Oseghe, S. Maddila, P.G. Ndungu, S.B. Jonnalagadda, Appl. Catal. B: Environ., 176 (2015)

288-297.

[31] J. Li, G. Lu, Y. Wang, Y. Guo, Y. Guo, J. Colloid Interf. Sci., 377 (2012) 191-196.

[32] J. Wang, S. Hou, L. Zhang, J. Chen, L. Xiang, CrystEngComm, 16 (2014) 7115-7123.

[33] I.Y. Bu, Ceram. Int., 39 (2013) 1189-1194.

[34] M.A. Mahmood, S. Baruah, J. Dutta, Mater. Chem. Phys., 130 (2011) 531-535.

[35] H. Zeng, G. Duan, Y. Li, S. Yang, X. Xu, W. Cai, Adv. Funct. Mater., 20 (2010) 561-572.

[36] Ü Özgür, Y.I. Alivov, C. Liu, A. Teke, M. Reshchikov, S. Do?an, V. Avrutin, S.-J. Cho, H. Morkoc, J. Appl. Phys., 98 (2005) 041301. 
[37] Y. Chen, D. Bagnall, H. J. Koh, K.T. Park, K. Hiraga, Z. Zhu, T. Yao, J. Appl. Phys., 84 (1998) 3912-3918.

[38] K. Vanheusden, C. Seager, W.t. Warren, D. Tallant, J. Voigt, Appl. Phys. Lett., 68 (1996) 403-405.

[39] K. Kotsis, V. Staemmler, Phys. Chem. Chem. Phys., 8 (2006) 1490-1498.

[40] I. Nakamura, N. Negishi, S. Kutsuna, T. Ihara, S. Sugihara, K. Takeuchi, J. Mol. Catal. A: Chem., 161 (2000) 205-212.

[41] X. Zou, J. Liu, J. Su, F. Zuo, J. Chen, P. Feng, Chem. Eur. J., 19 (2013) 2866-2873.

[42] Y. Zheng, C. Chen, Y. Zhan, X. Lin, Q. Zheng, K. Wei, J. Zhu, Y. Zhu, Inorg. Chem., 46 (2007) 6675-6682.

[43] A.B. Djurišić, Y.H. Leung, Small, 2 (2006) 944-961.

[44] T. Xu, L. Zhang, H. Cheng, Y. Zhu, Appl. Catal. B: Environ., 101 (2011) 382-387.

[45] P. Kundu, P.A. Deshpande, G. Madras, N. Ravishankar, J. Mater. Chem., 21 (2011) 4209-4216.

[46] Q. Wang, B. Geng, S. Wang, Environ. Sci. Technol,, 43 (2009) 8968-8973.

[47] W. Yao, B. Zhang, C. Huang, C. Ma, X. Song, Q. Xu, J. Mater. Chem., 22 (2012) 4050-4055.

[48] Z. Yi, J. Ye, N. Kikugawa, T. Kako, S. Ouyang, H. Stuart-Williams, H. Yang, J. Cao, W. Luo, Z. Li, Nat. Mater., 9 (2010) 559-564.

[49] F. Peng, Q. Zhou, D. Zhang, C. Lu, Y. Ni, J. Kou, J. Wang, Z. Xu, Appl. Catal. B: Environ., 165 (2015) 419-427.

[50] S. Liu, M. Q. Yang, Z. R. Tang, Y. J. Xu, Nanoscale, 6 (2014) 7193-7198. 


\section{Graphical abstract}
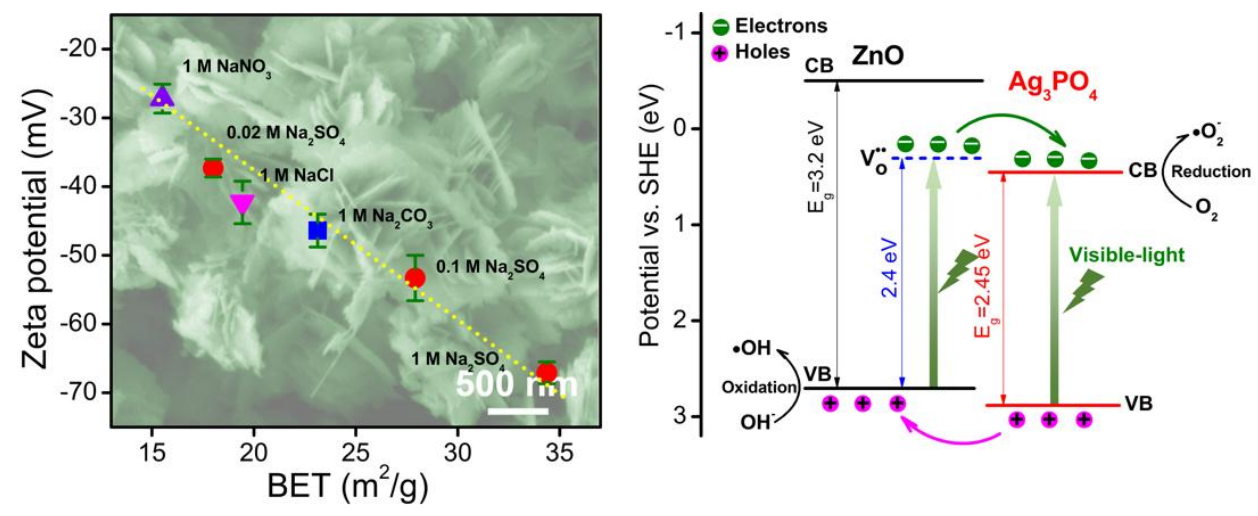

Defect-rich, high-surface-area $\mathrm{ZnO}$ nanosheets coupled with $\mathrm{Ag}_{3} \mathrm{PO}_{4}$ nanoparticles for efficient visible-light photocatalysis 